\title{
10 Rethinking consent in mHealth: (A) Moment to process
}

\author{
Iris Loosman
}

\section{Abstract}

The field of mobile health promises a transformation of the healthcare industry, by providing health-related information and services directly to individuals, through digital mobile devices. This presents society with new platforms for persuasive systems for healthy behavior change. Before such systems' full potential can be utilized, however, the question of how to consent to their use needs to be addressed. In this paper, I argue that one-off all-encompassing consent moments at the start of use of persuasive mobile health services do not suffice, given the functions they present, and the context in which they are used. Persuasive mobile health services are not only data-intensive, they are also designed to influence the user's behavior and health. Informed consent should be temporally distributed, in order to improve the quality of the user's autonomous authorization, that this context requires.

\subsection{Introduction}

Every day, hundreds of new mobile health applications enter the market. Many of these use persuasive techniques to initiate healthy behavior change for their users. Mobile health promises a transformation of the healthcare industry and is predicted to enable more personalized, participatory, preventive and less expensive care (Malvey and Slovensky 2014). Its systems bridge the clinical context on the one hand, with the context of everyday life on the other, through digital applications. One consequence of this bridging of contexts is the blurring of care norms and general information technology (IT) norms. As a result, the norm-based expectations that normally frame and ground informed consent to a treatment may not be clear, or may even be absent altogether (Voerman and Nickel 2017).

In this chapter I examine the need to rethink informed consent in mobile persuasive technologies for healthy behavior change. In particular, can the temporal distribution of informed consent improve autonomous authorization in these technologies? The chapter will be structured as follows. In section 1, I give a short overview of the relevant developments from the field. Sections 2 and 3 contain the main line of argumentation. Here I examine the features of mobile health apps more closely and argue that these features require improved autonomous authorization. I then expand on autonomous authorization and argue that its meaningful achievement requires appreciating informed consent as a process rather than a moment. Finally, I reflect on how these ideas can inform future design of informed consent in applications. I conclude this chapter with final remarks in the closing section. 


\subsection{Background}

Mobile health (henceforth: mHealth) is the practice of delivering health-related services to mobile phones and wearables. According to Oinas-Kukkonen and Harjumaa: "A growing number of information technology systems and services are being developed to change users' attitudes or behavior or both" (Oinas-Kukkonen and Harjumaa 2009 , p. 485). These systems can be especially useful within health contexts, for example to help prevent or manage chronic diseases, or keep healthcare costs down. The emergence of services designed to replace clinical treatments (so-called digital therapeutics), and services assisting patients with disease self-management, are two promising examples. Not only are these services ubiquitous and can involve artificial intelligence, they also collect extensive data with many potential uses, which are difficult to describe exactly and evaluate in advance. This makes it difficult to trust them and to consent to their use.

In this chapter informed consent is broadly taken to be the authorization of an activity, based on an understanding of what that activity entails, and absent of control by others (Grady 2015); in other words, an autonomous authorization (Faden and Beauchamp 1986). Faden and Beauchamp (1986) distinguish consent as autonomous authorization from legally or institutionally effective consent. Consent is effective when the procedure through which it is obtained satisfies the rules and requirements of a specific institutional practice (Faden and Beauchamp 1986). It is legally transformative, but not necessarily morally transformative (Edenberg and Jones 2019). Effective consent is often referenced in connection to (information) technology domains (Flick 2016; Edenberg and Jones 2019).

Debates on consent in data-intensive contexts reflect the difficulties of ensuring meaningful consent. Criticisms of the feasibility of achieving meaningful consent through ticking an "agree” box are common (Bashir et al. 2015; Custers 2016; Grady 2017; Ploug and Holm 2013). There are worries of consent desensitization, and routinisation - the amount of consent requests presented to users, together with the way these requests are set up, are not inviting users to engage the way they ideally should. Some authors argue this process might not be in line with what informed consent should ideally achieve:

\footnotetext{
Given the speed with which technology becomes integrated into our daily lives, often the values and expectations embedded in the technology itself become the default-whether or not we as a society have taken the time to think through the appropriate conditions for sharing personal information in exchange for access to digital services. It is easy to adopt as a given whatever terms of service are offered by the services we turn to on a daily basis. Individuals often feel powerless to negotiate these terms (Edenberg and Jones 2019, p. 1).
}

As a result, the resulting consent falls short of most normative standards of morally transformative consent (Edenberg and Jones 2019, p.1). Using an mHealth service is most often preceded by the step of downloading an application, followed by the dis- 
closure of Terms and Agreement, and a moment to click "agree". As mHealth is in essence an IT development relying on large dataflows, consent setups must adhere to data protection rules (Mantovani and Quinn 2013). However, a data protection perspective is not the only relevant perspective when looking at the quality of consent. mHealth has the potential to persuade consumers and patients alike (together: users), to achieve health-related goals, and to offer them treatment-like solutions. The bridging of health and general information technology contexts challenges traditional (often clinical) ideas about informed consent.

In this chapter I consider rethinking the informed consent often found in apps as a process, rather than a moment, with the goal of improving the quality of informed consent as an autonomous authorization. This implies that temporally distributed consent should offer a user multiple discrete consent moments over time; but in addition, it should also consider informed consent to be a process in which both the user and the technology develop over time.

\subsection{Rethinking consent in mHealth: Why, what and how?}

The current conception of consent in mHealth services is problematic for (at least) two reasons. The first can be described in terms of suboptimal user behavior-e.g. the user's clicking of "agree” without considering the consequences, without reading or fully grasping the Terms of Agreement. This behavior is a given, and is not properly addressed or mitigated by current consent setups (Bashir et al. 2015; Grady 2017). The second reason is that the current conception of consent shows insufficient consideration of the normative significance of the context of mHealth. The mobile services here discussed are capable of influencing users with potentially far-reaching consequences, which are supposed to be covered by a consent setup. Even in a world in which a user of mHealth behaved ideally, she still would not have meaningfully consented to all the relevant implications.

In section 2.1 I address these reasons under two themes: treatment (expectation) and persuasion. Here I discuss what I take to be normatively significant functions ${ }^{21}$ of mHealth, and the kind of consent these functions require. In section 3 I offer some thoughts on mitigating suboptimal user behavior in the design of consent processes.

21 In this chapter the term "function" is used to mean "intended purpose". 


\subsection{1 mHealth's functions}

\section{Treatment ${ }^{22}$ and expectations}

mHealth services are designed to perform functions that ethically require consent from their users: for example to monitor them, and to collect, share and store their data, whereby the extent and future purpose may often be unknown. These services provide the user with feedback in the form of knowledge, diagnoses and advice. This feedback is sometimes based on automated algorithms, without human oversight or the involvement of human judgment. These functions of mHealth can be viewed as counterparts of medical treatment, which would require a participatory action of the patient, within a clinical context.

Furthermore, many mHealth services, especially those that are direct-to-consumer, present themselves as a kind of therapy that improves health-related conditions (Martinez-Martin and Kreitmair 2018; Sax, Helberger and Bol 2018). The problem, according to Martinez-Martin and Kreitmair, is that "consumers may assume that their interactions with the service involve the kind of ethical obligations that are a part of professional therapy, making it particularly important to ensure that users understand that those obligations do not apply" (Martinez-Martin and Kreitmair 2018, p. 71). Looking at the treatment-like features of mHealth discussed above, it is not surprising that mHealth is often perceived this way. However, there are repercussions to misinterpreting what the system has to offer. There are risks associated with services that change health-related behavior, especially for users suffering from one or more diseases. Feedback, advice, or diagnoses can be wrong in general, or wrong for a particular patient, not taking her condition into account. Where a user expects to receive expert advice, this feedback is often the result of an algorithm, based on statistics, rather than one's personal circumstances. In that sense, using an mHealth service comes closer to self-diagnosis and self-treatment, with all the risks associated with those practices (Ruiz 2010).

A potential counterargument to this point is that because many of these services exist outside of medical practice, the responsibility is down to the user, and current consent setups fit with this assignment of responsibility. To this I would reply that responsibility-taking is only valid to the extent to which the user gets the opportunity to really understand what this means. Users of mHealth services must be facilitated to form reasonable expectations of what the systems actually offer, in order to meaningfully consent to their use.

Often the fine print of mHealth apps includes a statement regarding the non-medical nature of their services, in order to avoid liability. However, especially considering that mHealth apps are often sought by users suffering from health issues and seeking help, special attention should be paid to ensure that an appropriate overview

22 The term "treatment" is here used in a broad sense, to include diagnostics. 
of risks and benefits is provided (Martinez-Martin and Kreitmair 2018). Especially with the rise of digital therapeutics and assistive technologies, the vulnerability of potential users of mHealth services means extra attention must be paid to increasing understanding of what the app is, and what consenting to it entails. However, simply adding more information to the already top-heavy consent moment preceding use, is not making a real effort towards improving autonomous authorization.

\section{Persuasion}

The importance of consent in contexts of persuasion has been widely discussed (Oinas-Kukkonen and Harjumaa 2009; Spahn 2012; Timmer, Kool and van Est 2015). Spahn (2012), for example, argues that the user of such technology should always be informed about persuasion, and should give consent to being subjected to it. Emphasis is placed on ensuring that users are able to choose their own goals, and the methods of persuasion, in order to maintain respect for autonomy. With the developments of persuasive mHealth systems, to be downloaded directly onto users' personal mobile devices, the possibility for more data intensive data collection, and thus more personalization, opens up. According to Sax et al. (2018) it is to be expected that these personalization strategies will become more frequent and more powerful.

Oinas-Kukkonen and Harjumaa (2009) write that from an ethical point of view "it is necessary that the overall goal is made clear at all steps of incremental persuasion." They add: "Persuading a user is indeed a multi-phased and complex task, and different factors, such as the user's goal, may change during the process" (Oinas-Kukkonen and Harjuuma 2009, p.488). As a system learns and adjusts its methods to reach optimal results in behavior change, so too may a user of such a system change her motivation and goals. Can we reasonably expect a user of this technology to predict this at the initial moment of consent, and to understand what the unfolding persuasive strategies might entail?

The idea that the user's goals may change during the process of using mHealth apps is also emphasized by Sax et al. (2018) who identify three distinct stages of mHealth apps usage. These are: “(1) the decision to install an mHealth app; (2) the decision to start using an mHealth app; (3) and the decision to continue using mHealth apps for longer periods of time" (Sax et al. 2018, p. 114). The authors introduce this distinction to avoid "talking about 'the use of mHealth apps' as if this is a unitary phenomenon. At these different stages, different user motivations and different strategies to influence users can be observed" (Sax et al. 2018, p. 114).

Implementing one-off consent in mHealth services fails to acknowledge that different experiences and motivations can exist at each of the stages of mHealth usage. It is hard, if not impossible, to predict these beforehand, to understand what they entail, and to meaningfully decide. Sax et al. (2008) write: "Through usage, a sort of 'relationship' between user and app develops over time” (pp. 114-115). This relationship allows the app to make more accurate persuasive suggestions, but it could 
also mean that the user can more accurately judge whether or not this app matches expectations, for example. Compare a relationship with a physician, in which a bond of trust develops over time that can influence a patient's willingness to (continue to) consent. There, too, a patient can choose to alter or terminate her treatment, when her goals change, or when she or her physician determines it is not the best path of action. To facilitate a similar development in mHealth services, a user's informed consent should be able to develop over time.

\subsubsection{Improved autonomous authorization}

In the beginning of this chapter, I argued that current consent set-ups in mHealth services resemble what is described as effective consent more than autonomous authorization. However, because of suboptimal user behavior and insufficient addressing of mHealth's normatively significant features, neither sense of consent is taking place in a successful way. I also discussed several of mHealth's normatively significant features requiring improved consent. These features are related to treatment - on the level of the functions themselves, but also on the level of perceptions and expectations with regards to these functions - and related to persuasion. In what follows, I consider autonomous authorization in further detail, before moving on to presenting temporal distribution as a way to improve it.

According to Faden and Beauchamp (1986), informed consent as an autonomous authorization requires substantial understanding, substantial absence of control by others, intentionality, and an authorization of a professional to undertake a certain action. It is an effective communication of an intentional transfer of rights and obligations between parties, transforming the moral landscape between them, making actions permissible that otherwise were not (Edenberg and Jones 2019). But it does more than that. Consent in this sense is a way for a person to autonomously authorize a physician "to undertake diagnostic or therapeutic interventions" (Emanuel 2012, p. 5). That way, a patient can demonstrate understanding that she takes responsibility for her decision, while at the same time empowering another to implement it (Emanuel 2012).

Effective consent on the other hand, emphasises the written documentation of consent - disclosure, accompanied by a token of agreement. This is compatible with a more passive consent setup, in which the focus is on disclosing information and awaiting consent in return. Effective consent is not necessarily accompanied by autonomous decisions (Grady 2015), nor is it necessarily morally transformative. In order to facilitate the dynamic nature of decision-making, consent set-ups need to move beyond one-off, all-or-nothing decisions.

To illustrate this point in more detail, imagine a physician handing a book filled with terms and conditions for a certain procedure to a patient, leaving the room, and awaiting a token of informed consent in return. It is then up to the patient to make 
the information her own, to understand what risks and consequences the procedure will have, and to determine whether she trusts the physician with this procedure, for example. Let us assume the patient then chooses to go ahead with the procedure. In hindsight, it may turn out the patient may have overlooked or misunderstood that piece of crucial information that was so relevant for her. Perhaps the procedure has consequences for her practicing her favourite hobby. Perhaps it has consequences for her family, somehow. "Well, but you consented" the physician might say. This is in no way a realistic scenario for a clinical context. And yet this is what is expected of users of mHealth services, which, as I have discussed, contain many counterpart functions of clinical treatment, and can have far-reaching effects on health-related behavior.

To ensure that meaningful and morally transformative consent can take place in persuasive mHealth services, given the features discussed up until now, I argue the best way forward is to improve autonomous authorization, by temporally distributing consent in a developing process.

\subsubsection{Meaningful consent: Temporal distribution}

Thinking about temporal distribution of consent for mHealth requires the rethinking of informed decisions. Decisions are best understood as continuums or ongoing processes over time. The theory of distributed decision-making, found in psychology and sociology, supports this idea. According to Rapley (2008), decision-making is an ongoing event, distributed, amongst other dimensions, across time. Rapley describes that we could 'expand the idea of 'decisions' beyond the 'solo' cognitions and actions of individual, rational, autonomous human actors that occur in relatively atemporal and ahistorical consultations. Moving beyond our present static research focus on 'orphan' consultations, to a more distributed vision, may offer new opportunities to actively engage with, support and research decision-making-in-action” (Rapley 2008, p. 432).

Approaching informed consent as a static state in which a user takes the amount of time needed to review all information on offer, and comes to an informed decision, is unrealistic. Decision-making is something that takes place in action and over time (Rapley 2008). In line with this idea, I argue that a more dynamic process of consent, that is temporally distributed, has to satisfy (at least) three requirements. It should offer a user multiple discrete consent moments over time (1); and it should consider that both the user (2) and the technology (3) develop over time. I will briefly address these requirements in turn.

The first requirement, of offering multiple consent moments, would entail that a user or patient is able to start using a service with a low threshold of decision-making. Downloading and starting to use a service should be possible without first giving one's full and everlasting consent. However, merely distributing decisions over a number of discrete moments in time, is not going to improve the quality of consent. 
The act of offering information does not necessarily lead to informed decision-making (Manson and O'Neill 2007), no matter how many times it is asked of the user. This links to the second requirement.

Taking into account the gradual learning and the experience that is needed to make a decision, I suggest breaking the single consent moment in mHealth down into a process, and facilitate the learning process of the user. Thereby moving beyond multiple consent moments spread out over time, to ensure that the user is facilitated or equipped to make the relevant decisions, when they come up. As a user starts to learn more about the service through experience, about its functions and methods, so too can consent be required for more complex aspects. Not only does the user learn about the technology and what it entails through its use, she can also formulate new goals or expectations of using the technology. Being able to modify her decisions and change direction is key to facilitate this development.

To satisfy the third-mentioned requirement, a temporal distribution of consent must facilitate that not only the user is in constant flux, but the technology as well. As the user starts formulating certain goals and expectations, these could influence persuasive techniques employed by the service. Adjustments in the methods of persuasion, or for example in the purposes of data collection, mean informed consent should also be adjusted. As these aspects develop along the way and are hard to foresee and predict, they cannot be covered by a single decision in time.

These three requirements point towards a more interactive process, where consent interplays with both the user's as well as the service's development over time. Given the many technological possibilities that mHealth technologies provide, it might be possible to combine different kinds of consent within an innovative design. The precise content of this design would be topic for further research. ${ }^{23}$ Essential for mHealth is to ensure that the process is relevant for the individual user, giving her the opportunity to form a reasonable expectation, increasing understanding and intentionality.

Keeping the user and their vulnerabilities and uncertainties in mind is crucial in developing a consent process that can actually result in meaningful autonomous authorizations. Ultimately, the goal is not to increase quantity, but quality - to give users an opportunity to consent meaningfully. As persuasive mHealth apps are introduced in medical and non-medical contexts, to a wide range of users, from patients to lifestyle-focused consumers, this ethical hurdle should not be overlooked.

23 Examples of design ideas for other, related contexts can be found in the "dynamic consent" interface for biobanking (Kaye 2016), and "informed consent for information systems" (Friedman, Lin and Miller 2005). 


\subsection{Towards design}

A potential objection to the temporal distribution of informed consent can be found in the worry that too many consent transactions with too much (written) information could lead to consent routinization (Ploug and Holm 2015) and desensitization (Schermer, Custers and van der Hof 2014, pp. 176-7). Custers (2016) describes what happens with consent in online environments:

Due to the large number of consent requests, users often do not really consider the questions asked, do not read the information provided and do not seem to think through the consequences of providing (or refusing) consent. [...] This is obviously problematic, as such consent no longer has any meaning (Custers 2016, p. 3).

In other words, this could cause the suboptimal user behavior mentioned in the beginning of this chapter. Increasing the number of consent requests does not necessarily increase the meaningfulness of consent.

The meaningfulness of consent is not helped either by requiring an expert decision prior to even having opened the service. The very least that can be done is to ask for re-consent over the course of use. To this end, Custers (2016) introduces the idea of consent expiration dates and re-consent opportunities. Even though this approach acknowledges that there are elements involved in consent that might change over time, re-consent could potentially just be another checkbox for disengaged users (Custers 2016). Even though this does solve some problems with one-off consent, it does not necessarily make consent any more meaningful.

My response to these worries is that temporally distributing consent is not a quantitative change alone; it is crucially qualitative too. One of the goals of temporally distributing informed consent is to prevent the occurrence of consent overload and desensitization altogether. Providing users with stimulating informative materials, and actually supporting their decision-making processes, rather than overwhelming them with a bulk of information and sole responsibility for the consequences of their consent, are elements to incorporate in the design.

Not every instance of informed consent will be ideal, but with increased opportunities for autonomous authorization, there will be several benefits even for those who fall short of the ideal. First, there will be a general improvement in the realization of autonomous authorization. Even those who fall short of the ideal might improve how well they understand and relate to their treatment. Second, having the option to reconsider and reflect on consent, even if this option is not always exercised, improves the situation of the user by giving them the freedom (not) to exercise the option. And third, there will be side benefits from those who do approximate the ideal, for others who will not, by generating knowledge and expectations that spread. 


\subsection{Concluding reflections}

The goal of this chapter has been to open up the debate for meaningful consent in the mHealth context, given the many complexities and ethical issues it provides. mHealth is about more than data protection. It bears a resemblance with medical treatment, and has the potential of being introduced into medical settings. Given mHealth's features, and its possibilities of influencing its user, it is unrealistic to believe that this can be dealt with in a one-off all-encompassing decision. There is an ethical significance to mHealth services for health-related behavior change that needs to be addressed. A reconsideration of an ethical notion of consent, such as autonomous authorization, can inform this project.

In this chapter, I have argued that temporally distributed informed consent is an approach that could support the realization of this ethical notion of consent. However, as the title of this chapter suggests, the aim here has been to rethink consent in mHealth, rather than to offer a ready-made solution or design. The ideas posed here will need to be refined in light of empirical testing. As mHealth promises a transformation of the healthcare industry and is predicted to enable more personalized, participatory, preventive and less expensive care (Malvey and Slovensky 2014), this would be a worthwhile follow-up study. Given mHealth's promises, we should make sure that informed consent processes work in favor of achieving those ends.

\subsection{Acknowledgment and funding information}

This research is affiliated with the Netherlands Organisation for Scientific Research - Responsible Innovation (NWO-MVI) project "Mobile Support Systems for Behaviour Change," project number 100-23-616.

\section{References}

Bashir, Masooda, April D. Lambert, Carol Hayes, and Jay P. Kesan. 2015. Online Privacy and Informed Consent: The Dilemma of Information Asymmetry. ASIST. https://doi.org/10.1002/ pra2.2015.145052010043

Beauchamp, Thomas, and James Childress. 2013. Principles of Biomedical Ethics. $7^{\text {th }}$ ed. New York: Oxford University Press.

Custers, Bart. Click here to consent forever: Expiry dates for informed consent. 2016. Big Data \& Society. https://doi.org/10.1177/2053951715624935

Edenberg, Elizabeth, and Meg Leta Jones. 2019. Analyzing the legal roots and moral core of digital consent. New Media \& Society. https://doi.org/10.1177\%2F1461444819831321

Emanuel, Ezekiel J. 2012. Bioethics in the Practice of Medicine. Goldman's Cecil Medicine. https:// doi.org/10.1016/B978-1-4377-1604-7.00002-6

Faden, Ruth R., and Thomas Beauchamp. 1986. A history and theory of informed consent. New York: Oxford University Press. 
Flick, Catherine. 2016. Informed Consent and the Facebook emotional manipulation study. Research Ethics. https://doi.org/10.1177/1747016115599568

Friedman, Batya, Peyina Lin, and Jessica K. Miller. 2005. Informed Consent by Design. In Security and usability: Designing secure systems that people can use, eds. Lorrie Cranor and Simson Garfinkel, 495-521. Sebastopol: O'Reilly.

Grady, Christine. 2015. Enduring and emerging challenges of informed consent. The New England Journal of Medicine 372: 855-62.

Grady, Christine. 2017. The Changing Face of Informed Consent. The New England Journal of Medicine 376: 856-867.

Kaye, Jane, Edgar A. Whitley, David Lund, Michael Morrison, Harriet Teare, and Karen Melham. 2015. Dynamic Consent: A Patient Interface for Twenty-first Century Research Networks. European Journal of Human Genetics 23: 141-146.

Malvey, Donna, and Donna J. Slovensky. 2014. mHealth - Transforming Healthcare. New York: Springer.

Manson, Neil C., and O'Neill, Onora. 2007. Rethinking Informed Consent in Bioethics. New York: Cambridge University Press.

Mantovani, Eugenio, and Paul Quinn. 2013. mHealth and data protection - the letter and the spirit of consent legal requirements. International Review of Law, Computers \& Technology. https://doi.or $\mathrm{g} / 10.1080 / 13600869.2013 .801581$

Martinez-Martin, Nicole, and Karola Kreitmair. 2018. Ethical Issues for Direct-to-Consumer Digital Psychotherapy Apps: Addressing Accountability, Data Protection, and Consent. JMIR Mental Health. https://doi.org/10.2196/mental.9423

Oinas-Kukkonen, Harri, and Marja Harjuuma. 2009. Persuasive Systems Design: Key Issues, Process Model, and System Features. Communications of the Association for Information Systems 24: 485-500.

Ploug, Thomas, and Søren Holm. 2013. Informed consent and routinization. Journal of Medical Ethics 39: 214-218.

Rapley, Tim. 2008. Distributed decision making: the anatomy of decisions-in-action. Sociology of Health \& Illness, 3, 429-444.

Ruiz, María E. 2010. Risks of self-medication practices. Current Drug Safety. 5: 315-23.

Sax, Marijn, Natali Helberger, and Nadine Bol. 2018. Health as a Means Towards Profitable Ends: mHealth Apps, User Autonomy, and Unfair Commercial Practices. Journal of Consumer Policy. https://doi.org/10.1007/s10603-018-9374-3

Schermer, Bart W., Bart Custers, and Simone van der Hof. 2014. The Crisis of Consent: How Stronger Legal Protection May Lead to Weaker Consent in Data Protection. Ethics and Information Technology $16: 171-182$.

Spahn, Andreas. 2012. And lead us not into persuasion? Persuasive technology and the ethics of communication. Science and Technology Ethics 18, 4: 633-650. doi:10.1007/s11948-011-9278-y

Timmer, Jelte, Linda Kool, and Rinie van Est. 2015. Ethical Challenges in Emerging Applications of Persuasive Technologies. PERSUASIVE 2015 (Conference Proceedings): 196-201.

Voerman, Sander A., and Philip J. Nickel. 2017. Sound trust and the ethics of telecare. Journal of Medicine and Philosophy. https://doi.org/10.1093/jmp/jhw035 
